\title{
Optically oriented and detected electron spin resonance in a lightly doped n-GaAs layer
}

John S. Colton

john_colton@byu.edu

\section{T. A. Kennedy}
A. S. Bracker
D. Gammon

J. B. Miller

Follow this and additional works at: https://scholarsarchive.byu.edu/facpub

Part of the Astrophysics and Astronomy Commons, and the Physics Commons

\section{Original Publication Citation}

Optically oriented and detected electron spin resonance in a lightly doped n-GaAs layer, J.S. Colton, T.A. Kennedy, A.S. Bracker, D. Gammon, and J.B. Miller, Phys. Rev. B 67, 165315 (23). The original version may be found at: http://prb.aps.org/abstract/PRB/v67/i16/e165315

\section{BYU ScholarsArchive Citation}

Colton, John S.; Kennedy, T. A.; Bracker, A. S.; Gammon, D.; and Miller, J. B., "Optically oriented and detected electron spin resonance in a lightly doped n-GaAs layer" (2003). Faculty Publications. 503. https://scholarsarchive.byu.edu/facpub/503 accepted for inclusion in Faculty Publications by an authorized administrator of BYU ScholarsArchive. For more information, please contact ellen_amatangelo@byu.edu. 


\title{
Optically oriented and detected electron spin resonance in a lightly doped $\boldsymbol{n}$-GaAs layer
}

\author{
J. S. Colton, ${ }^{*}$ T. A. Kennedy, A. S. Bracker, D. Gammon, and J. B. Miller \\ Naval Research Laboratory, Washington, D.C. 20375 \\ (Received 28 October 2002; revised manuscript received 11 December 2002; published 24 April 2003)
}

\begin{abstract}
Spin resonance of localized electrons bound to donors in a specially designed $n$-GaAs layer has been performed at $236 \mathrm{MHz}$ and $41 \mathrm{mT}$, using circular polarized light to polarize the electrons and photoluminescence to detect the electronic polarization. The polarization was diminished under the resonance condition. The electronic $g$ factor obtained by this measurement is $-0.41 \pm 0.01$. The resonance linewidth of $2 \mathrm{mT}$ corresponds to a spin lifetime of $28 \mathrm{~ns}$. In order to observe the electronic spin resonance, nuclear effects were eliminated by application of rf fields to simultaneously resonate the nuclear spins.
\end{abstract}

DOI: 10.1103/PhysRevB.67.165315

PACS number(s): 76.30.-v, 78.55.Cr, 72.25.Rb, 72.25.Fe

The study of spin in semiconductors has taken on increased importance with potential applications involving quantum computing, such as the proposal to use electronic spin in a quantum dot as a scalable qubit. ${ }^{1}$ Recent exciting measurements of long electronic spin lifetimes in $n$-GaAstimes of 42 ns (Ref. 2), 130 ns (Ref. 3), and 300 ns (Ref. 4) - support the quantum dot approach. These lifetimes exceed the optical recombination time, as well as the hyperfine interaction-limited spin lifetime of $1-5 \mathrm{~ns}$ expected for an electron in a quantum dot. ${ }^{4-6}$ The longer spin lifetimes have been obtained as a result of spin existing in the excess electrons, and some electron delocalization made possible through hopping between donors. ${ }^{7,8}$

Due to the strong hyperfine interaction in III-V compounds, most of the previous magnetic resonance in GaAs has been performed on conduction electrons. In the conduction-band electron magnetic resonance (CESR) of Weisbuch and Hermann, 9,10 the electrons were polarized optically and the resonance was detected through a change in the optical polarization of the photoluminescence (PL). Optically polarized and optically detected magnetic resonance in GaAs was extended into heterostructures (including quantum wells) with higher resonant fields, although still in $p$-type or nominally undoped material. ${ }^{11,12}$ There have also been a large number of papers on electrically detected resonance in two-dimensional electron gases, with the most applicable to this work being an experiment on a GaAs heterostructure involving the combined effects of electron spin resonance (ESR) and nuclear magnetic resonance (NMR). ${ }^{13}$ The only instance of magnetic resonance on more localized electrons in lightly $n$-doped $\left(n<10^{16} \mathrm{~cm}^{-3}\right)$ samples has been conventional ESR of an $n \approx 10^{15} \mathrm{~cm}^{-3}$ sample at high fields $(6-10 \mathrm{~T}){ }^{14}$

Electrons bound to donors in lightly doped $n$-type samples model electrons localized in quantum dots. We report an observation of optically polarized and optically detected magnetic resonance of electrons bound to donors in an $n=3$ $\times 10^{15} \mathrm{~cm}^{-3} \mathrm{GaAs}$ layer. These electrons approach the localized limit but maintain a slight delocalization and extended lifetime, which has assisted in making the resonance visible. Important aspects to our observation have been the design of sample, the choice of resonant frequency, the optical polarization of electrons, and the control of nuclear spins. The electrons have a measured $g$ factor of -0.41 and a linewidth of $2 \mathrm{mT}$, which corresponds to a spin lifetime of $28 \mathrm{~ns}$.

The experiments described in this paper have been performed on a specially designed MBE $n$-GaAs sample. The active layer was $1 \mu \mathrm{m}$ thick and doped at a nominal 3 $\times 10^{15} \mathrm{~cm}^{-3}$ level. The sample was of high quality and had very bright PL. The active layer was surrounded by AlGaAs layers and designed primarily with three considerations in mind. First, the AlGaAs layers reduce unwanted nonradiative surface recombination. Second, the doping levels were chosen to minimize band-bending effects and place the Fermi level near the conduction-band minimum. Third, the AlGaAs layers prevent spin diffusion into the bulk, which can occur at length scales much larger than the $1-\mu \mathrm{m}$ absorption length. ${ }^{2}$ More details on the sample preparation can be found elsewhere. ${ }^{15}$

A relatively low frequency $(236 \mathrm{MHz})$ at which to perform the electron resonance, with a correspondingly low resonant field, was chosen based on a number of considerations. A low-field experiment minimizes the effects of $g$ broadening which can be large at high fields (the resonant linewidth was broadened to $50 \mathrm{mT}$ in the previously mentioned high-field ESR experiment ${ }^{14}$ ), minimizes the effects of diamagnetic PL peak position shift with field and should offer some ability to extend resonance experiments into samples with smaller $g$ factors such as GaAs quantum wells and quantum dots. ${ }^{16}$ The low resonant field means that polarization of electrons must be done optically rather than thermally - an added advantage, in that the holes do not become polarized as they would under high magnetic fields, making it possible to get a clear signature of the electrons.

Optical polarization of electronic spin is performed via excitation with circularly polarized light. This is the standard technique of "optical orientation." "10 The excitation wavelength was $785 \mathrm{~nm}(1.579 \mathrm{eV})$, which is between the bandgap energy and the split-off valence band as is necessary in the optical orientation technique. It was modulated at $20 \mathrm{kHz}$ with a photoelastic modulator (PEM) operating as an oscillating quarter wave plate, to produce alternating circular polarizations $\sigma^{+}$and $\sigma^{-}$. Modulation of the incident light is necessary to minimize nuclear polarization. The PL was collected, analyzed for circular polarization, passed through a SPEX 1680 0.22-m double-grating spectrometer and detected with a photomultiplier tube-photon counting system 


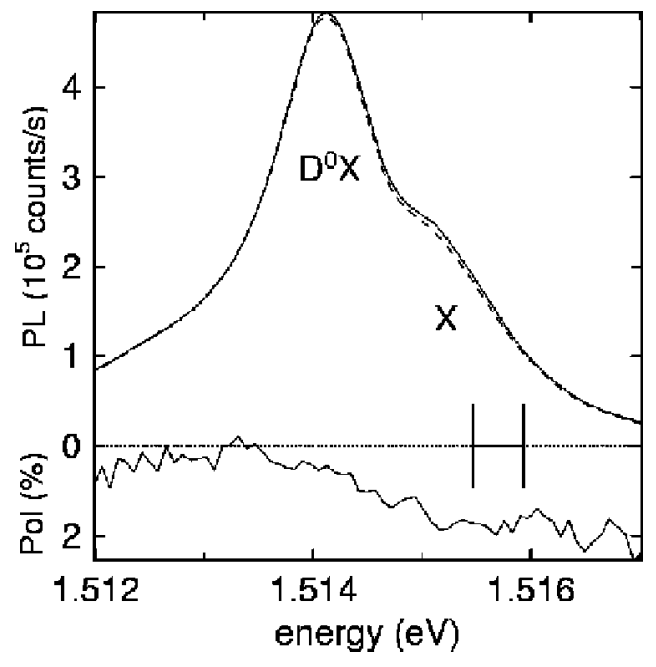

FIG. 1. Upper: $1.5 \mathrm{~K}$ photoluminescence for $\sigma^{+}$and $\sigma^{-}$excitation. Lower: polarization deduced from $\left(\sigma^{+}-\sigma^{-}\right) /\left(\sigma^{+}+\sigma^{-}\right)$. The spectrometer resolution and PL detection energy for the resonance experiments are marked by the bars.

which operated synchronously with the PEM to detect the effect of the two incident polarizations independently. All of the experiments were performed in an Oxford liquid-helium cryostat-superconducting magnet system, at either $1.5 \mathrm{~K}$ (pumped helium) or $6 \mathrm{~K}$ (gas flow cooling).

The electronic polarization was monitored through the optical polarization of photoluminescence. The effects of $\sigma^{+}$ and $\sigma^{-}$excitation are seen for a representative PL spectrum in Fig. 1. These data and those of our magnetic resonance were taken with an excitation power density of $2 \mathrm{~W} / \mathrm{cm}^{2}$, chosen for this sample such that the electron lifetime $\left(\tau_{e l}\right.$ $=n / G$ where $G=$ generation rate of photoexcited electrons) was slightly longer than the spin dephasing time. ${ }^{15}$ This may be compared with the typical light levels of $100 \mathrm{~W} / \mathrm{cm}^{2}$ in the Weisbuch-Hermann CESR. ${ }^{10}$ The concentration of photoexcited excitons at $2 \mathrm{~W} / \mathrm{cm}^{2}$ is approximately $5 \times 10^{13} \mathrm{~cm}^{-3}$, much smaller than the concentration of doped electrons. Both the free-exciton and the donor-bound exciton are apparent. The donor-bound exciton peak was unpolarized, as expected, because in the singlet state at the time of recombination there are two electrons present having opposite spin. The PL of the free exciton, however, did become polarized, and the polarization increased with increasing excitation power density up to some maximum value, as expected. ${ }^{15}$

In $n$-type material, it has long been known that the polarization of emission of the free exciton can be indicative of the polarization of the doped electrons. ${ }^{17}$ Paget found that spin exchange between nonlocalized photoelectrons and localized doped electrons is very fast, on the order of tens of picoseconds, and provides an efficient averaging of spin between the two types of electrons. ${ }^{18}$ Thus, when the photoexcitation injects spin-polarized electrons, these relax to the bottom of the conduction band in a largely spin-conserving process and then interact with the doped electrons via exchange to polarize the doped electrons. The electrons in excitons will likewise become spin polarized, and this will

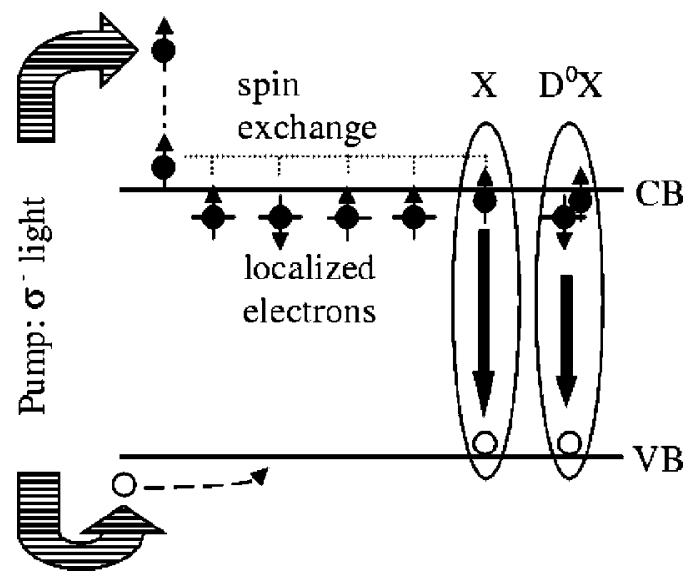

FIG. 2. Schematic diagram of the polarization process. Circularly polarized light injects spin-polarized electrons and unpolarized holes. The electrons relax in a largely spin-conserving manner to the bottom of the conduction band (CB). The exchange interaction continually equilibrates spin between the nonlocalized photoexcited electrons and the localized donor electrons. The electrons in free excitons become spin polarized, and the free exciton displays polarized luminescence. Emission from the donor-bound exciton is at a slightly lower energy and unpolarized. Changes to the polarization of the electron system are displayed in the luminescence of the free excitons.

cause the excitonic luminescence to be polarized. The interaction between photoexcited electrons, luminescent excitons, and the reservoir of doped electrons has been used by many groups to obtain spin information from the exciton luminescence, ${ }^{2,4,8,15,17,18}$ despite the exciton lifetimes themselves being about 1 ns. ${ }^{19}$

Implicit in this description is a lack of polarization of the holes, which is the case due to their much more rapid spin relaxation. This process is shown schematically in Fig. 2 . Any change of the polarization of the electron system will be reflected in the exciton luminescence. A magnetic resonance may thus be observed as a decrease in the PL polarization, similar to other experiments mentioned above. ${ }^{9,11,12}$ Again, this is only possible through the selection rules which connect the optical polarization to the electronic spin.

As mentioned above, magnetic resonance experiments in $n$-type GaAs are made more difficult due to hyperfine interaction with the nuclei. Any departure of the electron polarization from thermal equilibrium causes the electrons to attempt to relax through the hyperfine interaction and produces a dynamic polarization of the nuclei. This is the Overhauser effect. Electrons in our system are taken out of equilibrium via optical pumping and also via electron spin resonance. When the nuclei become polarized, they produce an "Overhauser shift" of the electron resonance peak due to an effective magnetic field. Theoretical estimates of the time scale in which the nuclei become polarized are from $10^{4} \mathrm{~s}$ (interactions with free electrons) to $0.1 \mathrm{~s}$ (localized electrons), ${ }^{10}$ and experimental measurements have been 1-10 s (electrons in GaAs quantum $\operatorname{dots}^{20}$ ) and $1 \mathrm{~s}$ (electrons in a $n=7$ $\times 10^{14} \mathrm{~cm}^{-3}$ bulk GaAs sample). ${ }^{14}$ The Overhauser effect was addressed in our experiment through performing nuclear 


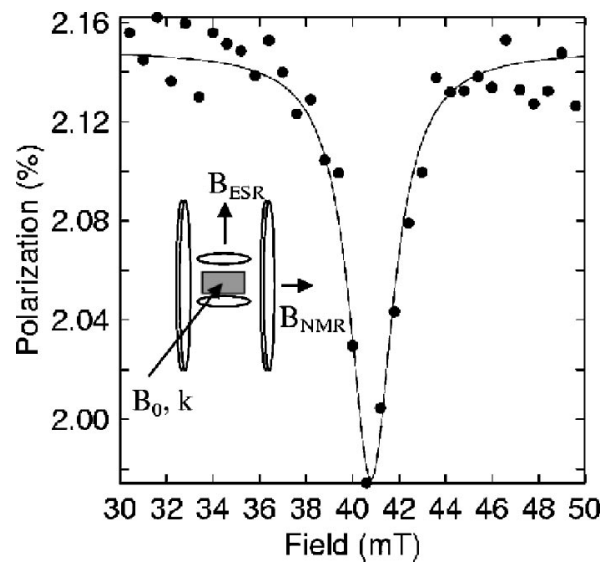

FIG. 3. Magnetic resonance spectrum under representative conditions, fitted to a Lorentzian with $|g|=0.41$ and $\Delta B=2.3 \mathrm{mT}$. The optical power density was $2 \mathrm{~W} / \mathrm{cm}^{2}$, and the temperature was $6 \mathrm{~K}$. The ESR power was $20 \mathrm{~W}$. The NMR power was $20 \mathrm{~W}$ and was swept from 220 to $650 \mathrm{kHz}$ at a repetition rate of $1 \mathrm{~Hz}$. Inset: a schematic drawing of the sample chamber (sample is shaded rectangle), showing the directions of the optical $\mathbf{k}$ and the three magnetic fields.

resonance simultaneously with the electron resonance.

The sample was placed in a Teflon holder at the center of a one-turn Helmholtz coil $(1 \mathrm{~cm}$ diameter $)$ in series with a capacitance of $2.6 \mathrm{pf}$, to give a resonant circuit at $236 \mathrm{MHz}$. Two additional capacitors were added in parallel with the resonant circuit to provide impedance matching to $50 \Omega .^{21}$ The circuit provided an oscillating magnetic field perpendicular to the static longitudinal field, with an amplitude of $B_{E S R}=2.0 \mathrm{mT}$ with $20 \mathrm{~W}$ of VHF power. An additional sixturn Helmholz coil (2.5 $\mathrm{cm}$ diameter) was set perpendicular to both the $236 \mathrm{MHz}$ coil and the longitudinal field, which applied $\mathrm{rf}$ waves for resonance at the nuclear frequencies. The second coil applied a magnetic field with an amplitude of approximately $B_{N M R}=0.8 \mathrm{mT}$ with $20 \mathrm{~W}$ of rf power over the frequencies of interest. The $\mathrm{rf}$ was scanned repetitively from 220 to $650 \mathrm{kHz}$ at a rate of $1 \mathrm{~Hz}$ (chosen as optimal after a preliminary study), and caused the three nuclear isotopes $\left({ }^{71} \mathrm{Ga},{ }^{69} \mathrm{Ga}\right.$, and $\left.{ }^{75} \mathrm{As}\right)$ to be sequentially saturated. This technique of scanning through NMR frequencies has been used previously in GaAs quantum dots. ${ }^{20} \mathrm{~A}$ schematic of the sample holder showing the static magnetic field and coils is shown in the inset to Fig. 3.

A change of PL polarization was observed as the magnetic field was swept through the ESR condition. All field sweeps were performed at rate of $1.6 \mathrm{G} / \mathrm{s}$, from high field to low field, and the data were averaged over many scans. Results are displayed in Fig. 3 for $20 \mathrm{~W}$ of VHF power, $20 \mathrm{~W}$ of $\mathrm{rf}$ power, and at $\mathrm{T}=6 \mathrm{~K}$. The resonance is observed as a decrease in luminescence polarization from $2.15 \%$ to $1.98 \%$. The resonance is seen at $41 \mathrm{mT}$, which corresponds to a $g$ factor of $-0.41 \pm 0.01$ according to the magnetic resonance equation $h \nu=g \mu_{B} B$, the negative sign being taken from the literature. The half-width of $1.1 \mathrm{mT}$ is slightly narrower than the Hanle half-width measured for this sample at this excitation power density (1.5 $\mathrm{mT}$ ) (Ref. 15) and corresponds to a spin lifetime of $28 \mathrm{~ns}$.
The electron $g$ factor measured by this experiment is slightly lower in magnitude than values reported in previous experiments. The $g$ factor measured for GaAs in the $p$-type samples of the CESR experiments was $-0.44 .{ }^{9}$ The values measured for the structures in Refs. 11 and 12 were in the range of -0.46 to -0.48 . The value obtained by Seck et al. for $n$-type GaAs was -0.464 , when the measured high-field values are extrapolated back to $0 \mathrm{~T}^{14}$ The value measured by Ostreich et al. in nominally undoped GaAs, via the "spin quantum beat" method, was $-0.44 .{ }^{22}$ The difference between these values and our own measurement likely comes from differences in degree of localization and doping. For example, the time resolved Faraday rotation data of Kikkawa and Awschalom show $|g|$ values of $0.43,0.45,0.14$, and 0.20 for GaAs samples that had $n=$ nominally undoped, $10^{16} \mathrm{~cm}^{-3}, 10^{18} \mathrm{~cm}^{-3}$, and $5 \times 10^{18} \mathrm{~cm}^{-3}$, respectively. ${ }^{3}$

For large rf powers, such as the conditions for Fig. 3, the nuclear polarization is "reset" to zero at a rate of $1 \mathrm{~Hz}$, and the Overhauser process is not fast enough to polarize the nuclei between sweeps. Thus there is no Overhauser shift, and on the time scale of the electrons, the nuclear spins are frozen. The line broadening is then very similar to the case of the Hanle experiment mentioned above, where the randomly oriented nuclear spins produce a random "frozen field" for each electron-the difference is the presence of a longitudinal magnetic field. In the zero-field case, the dephasing arises through electron precession in the random nuclear fields. Merkulov et al. discuss dephasing of localized electrons in quantum dots in the presence of a large longitudinal field and conclude that the frozen fluctuation model is still applicable, with the modification that the nuclear fields become oriented parallel and antiparallel to the longitudinal field so that the dephasing arises due to a distribution in field amplitudes rather than a distribution in field directions. ${ }^{6}$ It is therefore not surprising that the spin lifetime measured in this experiment is close to the Hanle lifetime.

The resonance peak showed a marked dependence on rf power. When no rf power was applied or if the scanning was not performed, the electron resonance could not be seen at all. This is very different from the CESR results on $p$-type GaAs, where CESR was still observed even when the nuclei were not resonant. These stronger nuclear effects are evidence that our resonance is arising from the localized electrons. When the rf was applied at lower power than in Fig. 3, the peak was broader, was shifted to a higher magnetic field, and was reduced in amplitude. These results are displayed in Fig. 4.

The change in peak position is an Overhauser shift. Since the peak is observed in the downward field scan before the normal resonance condition is met, the shift in peak position to higher fields must arise from a background nuclear polarization. Although a first step in eliminating a background Overhauser shift was taken by our use of a PEM in the excitation beam, a remnant nuclear polarization could be caused by a slight asymmetry in the polarization modulation or by a lack of perfectly circularly polarized excitation light - which is certainly the case, since the PEM produces a sinusoidal retardance modulation rather than a square-wave modulation. The change in peak width and amplitude also 


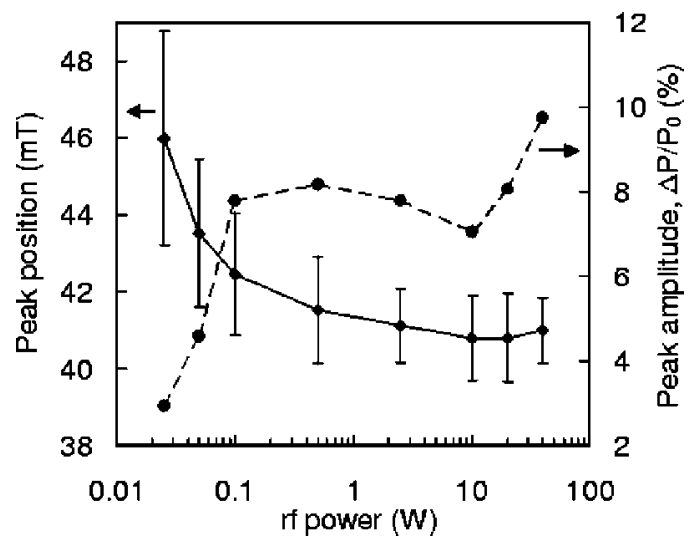

FIG. 4. rf power dependence of the resonance peak. Left axis: peak position, with peak width plotted as bars. Right axis: peak amplitude. For rf power less than $\sim 1 \mathrm{~W}$, Overhauser effects become prominent.

arises from an Overhauser shift. As the field sweep causes the electrons to come into resonance, the nuclear polarization begins to change. This shifts the resonance peak position and broadens the line. In the limit of no rf power, the line is broadened beyond observability. A similar effect has been seen by Hillman and Jiang in their studies of a GaAs $2 \mathrm{DEG},{ }^{13}$ in which case the ESR peak disappeared from the normal position, then reappeared as the Overhauser shift was eliminated due to application of nuclear resonance. Thus nuclear fields play two important roles at low rf powers: (a) a remnant nuclear field causes the peak position to be at higher fields and (b) a changing nuclear field due to changing electron polarizations during the ESR condition broadens the peak.

One question which remains is why the observed $\Delta P / P$ is lower than expected. One would expect the peak amplitude to depend strongly on the saturation factor. Since this resonance was performed under conditions where the $B_{E S R}$ is approximately equal to the resonance width, the saturation factor is nearly $100 \%$, and one should expect nearly all of the spins to be depolarized; i.e., $\Delta P / P$ should be close to $100 \%$ instead of the measured $8 \%-10 \%$. This may be evidence that even in the high-rf-power regime, we are still not completely eliminating nuclear effects.

The success of this experiment has been in finding conditions under which a clear electron resonance from the localized electrons in $n$-GaAs could be seen, which had not previously been done. Further investigation may help to clarify and quantify some of the important effects that have been observed. In particular, varying the direction and rate of the magnetic field sweeps could give more information on the Overhauser effect, as could perhaps varying the rf sweep rate for applying the nuclear resonance. A preliminary study of the ESR power led us to believe that no line broadening due to electron saturation effects was taking place, but that should perhaps also be investigated more closely.

In conclusion, we have made an observation of magnetic resonance in an $n$-GaAs sample through optical polarization of spins and optical detection of the spin polarization. The resonance occurred at $|g|=0.41$, and the $1 \mathrm{mT}$ half-width corresponds to a spin lifetime of $28 \mathrm{~ns}$. This manipulation of the spin of the localized doped electrons is precisely what must be accomplished for quantum computing schemes involving localized electronic spins. Nuclear effects through the hyperfine interaction have been shown to play a prominent role in the resonance process. The optical nature of our experiment means that extensions can be made into quantum wells and dots, where conventional absorption ESR is not possible due to the reduced number of spins. Another focus of our effort is an extension to a time domain experiment. The detection of spin resonance done in this work is a necessary precursor to coherent manipulations such as those performed in a spin echo experiment. Current theory predicts that the homogeneous $T_{2}$ in GaAs as revealed in a spin-echo experiment should be much longer than any of the inhomogeneous spin relaxation times that have been currently measured. ${ }^{6}$ Such an observation would be extremely important.

The authors would like to acknowledge and thank J.G. Tischler and Al.L. Efros for helpful and insightful discussions. J.S.C. is supported by NRL and NRC. Work has also been supported by DARPA and ONR.
*Electronic address: colton@bloch.nrl.navy.mil

${ }^{1}$ D. Loss and D.P. DiVincenzo, Phys. Rev. A 57, 120 (1998).

${ }^{2}$ R.I. Dzhioev, B.P. Zakharchenya, V.L. Korenev, and M.N. Stepanova, Phys. Solid State 39, 1765 (1997).

${ }^{3}$ J.M. Kikkawa and D.D. Awschalom, Phys. Rev. Lett. 80, 4313 (1998).

${ }^{4}$ R.I. Dzhioev, V.L. Korenev, I.A. Merkulov, B.P. Zakharchenya, D. Gammon, A.L. Effros, and D.S. Katzer, Phys. Rev. Lett. 88, 256801 (2002).

${ }^{5}$ M.I. D’yakonov and V.I. Perel', Sov. Phys. JETP 38, 177 (1974).

${ }^{6}$ I.A. Merkulov, A.L. Efros, and M. Rosen, Phys. Rev. B 65, 205309 (2002).

${ }^{7}$ K.V. Kavokin, Phys. Rev. B 64, 075305 (2001).

${ }^{8}$ R.I. Dzhioev, K.V. Kavokin, V.L. Korenev, M.V. Lazarev, B.Ya. Meltser, M.N. Stepanova, B.P. Zakharchenya, D. Gammon, and
D.S. Katzer, Phys. Rev. B 66, 245204 (2002).

${ }^{9}$ C. Weisbuch and C. Hermann, Phys. Rev. B 15, 816 (1977).

${ }^{10}$ Optical Orientation, edited by F. Meier and B.P. Zakharchenya (North-Holland, Amsterdam, 1984).

${ }^{11}$ M. Schreiner, H. Pascher, G. Denninger, and S.A. Studenikin, Solid State Commun. 102, 715 (1997).

${ }^{12}$ M. Krapf, G. Denninger, H. Pascher, and G. Weimann, Solid State Commun. 74, 1141 (1990).

${ }^{13}$ C. Hillman and H.W. Jiang, Phys. Rev. B 64, 201308 (2001).

${ }^{14}$ M. Seck, M. Potemski, and P. Wyder, Phys. Rev. B 56, 7422 (1997).

${ }^{15}$ J.S. Colton, T.A. Kennedy, A.S. Bracker, and D. Gammon, Phys. Status Solidi B 233, 445 (2002).

${ }^{16}$ A. Malinowski and R.T. Harley, Phys. Rev. B 62, 2051 (2000).

${ }^{17}$ V.B. Vekua, R.I. Dzhioev, B.P. Zakharchenya, and V.G. Fleisher, 
Sov. Phys. Semicond. 10, 210 (1976).

${ }^{18}$ D. Paget, Phys. Rev. B 24, 3776 (1981); 25, 4444 (1982).

${ }^{19}$ G.D. Gilliland, Mater. Sci. Eng., R. 18, 99 (1997).

${ }^{20}$ S.W. Brown, T.A. Kennedy, and D. Gammon, Solid State Nucl. Magn. Reson. 11, 49 (1998).
${ }^{21}$ J.B. Miller, B.H. Suits, A.N. Garroway, and M.A. Hepp, Concepts Magn. Reson. 12, 125 (2000).

${ }^{22}$ M. Oestreich, S. Hallstein, and W.W. Ruhle, IEEE J. Sel. Top. Quantum Electron. 2, 747 (1996). 